\title{
Nordiques
}

36 | 2018

Réformer l'éducation en Europe du Nord

\section{De la planification à la liberté de choix : une révolution pour le modèle éducatif suédois? Conversation avec Ulf P. Lundgren}

Piero Colla et Tomas Wedin

\section{(2) OpenEdition \\ Journals}

Édition électronique

URL : http://journals.openedition.org/nordiques/534

DOI : 10.4000/nordiques.534

ISSN : 2777-8479

Éditeur :

Association Norden, Bibliothèque de Caen la mer

\section{Édition imprimée}

Date de publication : 1 novembre 2018

Pagination : 115-128

ISBN : 9791095914020

ISSN : 1761-7677

\section{Référence électronique}

Piero Colla et Tomas Wedin, « De la planification à la liberté de choix : une révolution pour le modèle éducatif suédois? Conversation avec Ulf P. Lundgren », Nordiques [En ligne], 36 | 2018, mis en ligne le 31 octobre 2020, consulté le 13 mars 2021. URL : http://journals.openedition.org/nordiques/534 ; DOI : https://doi.org/10.4000/nordiques.534 


\section{De la planification à la liberté de choix : une révolution pour le modèle éducatif suédois ? Conversation avec Ulf P. Lundgren}

Monsieur Lundgren, depuis la fin des années 1960 - lorsque la nouvelle école universelle, la grundskola, venait de se généraliser à l'échelle du pays -, vous êtes au centre des débats sur les enjeux de la modernisation et de la gouvernance du système scolaire suédois. Mis à part vos fonctions de professeur de pédagogie à l'université d'Uppsala et d'expert pour un grand nombre de projets et enquêtes publiques, vous avez exercé de hautes responsabilités dans l'appareil administratif : président du Läroplanskommitté (1991-1992), qui a été responsable de la révision des curricula de 1993-1994, et surtout directeur général de Skolverket, l'Agence nationale de l'éducation, pendant les neuf premières années de son existence, de 1991 à 1999.

Depuis cette époque, le système éducatif suédois a considérablement évolué sur le plan quantitatif : l'intégration de l'école maternelle dans l'architecture du système et l'investissement dans l'éducation des adultes en ont fait monter les effectifs d'environ 1220000 enfants et 200000 adultes en 1993 à un total de 2,5 millions, toutes catégories confondues, en $2017^{1}$. À cela s'ajoute l'impact de l'immigration, dû aux flux de réfugiés politiques qui ont investi la Suède depuis les années 1980, et qui a connu une accélération considérable au cours des années 2015-2016. La mixité ethnique, culturelle et linguistique - phénomène marginal jusqu'aux années 1970 - est désormais une réalité à tous les échelons du système. Mais c'est surtout au niveau de ses principes directeurs que le système a connu un changement de paradigme : d'après de nombreux observateurs, certains éléments caractéristiques du modèle éducatif suédois (primat de l'intérêt public, recherche de l'égalité, rôle de l'expertise pédagogique dans la planification des politiques) ont perdu leur poids. Selon les mots de Tomas Englund, sociologue à Örebro, l'école se serait transformée d'un "bien public » en un "bien privé ${ }^{2}$. La transition serait illustrée par la possibilité reconnue à chaque élève de choisir son école et par la dérégulation de l'établissement des écoles privées, qui connaissent, depuis la fin des années 1990, un développement prodigieux : elles constituent

1 Source : Skolverket.se/statistik, 2017.

2 Tomas Englund, "Utbildning som "public good” eller "private good” ? ", in Utbildningspolitiskt systemskifte ?, T. Englund (dir.), Stockholm, HLS Förlag, 1996. 
$27 \%$ des maternelles, $17 \%$ des écoles de base, et $33 \%$ des lycées ${ }^{3}$. La Suède est aujourd'hui l'un des pays où la marchandisation du système éducatif est la plus développée dans le monde ${ }^{4}$.

Alors que vous étiez aux commandes de Skolverket, les principales réformes qui ont traduit cette révolution en pratique ont vu le jour : dévolution aux municipalités des responsabilités de gestion et du recrutement des enseignants (1989), un système de " chèques-éducation " - ou vouchers ${ }^{5}$ - pour l'accès à l'enseignement privé et l'introduction d'une forme de direction par objectifs et résultats à atteindre, plutôt que par des règles détaillées. Vous êtes donc un témoin privilégié de ce tournant, mais vous êtes également familiarisé - à travers votre engagement international et vos responsabilités à l'OCDE ${ }^{6}$ - avec les sources d'inspiration de ces réformes. Par votre rôle lors de la mise en place du système d'évaluation PISA (2000), vous avez été à la pointe de l'émergence d'une nouvelle culture de l'évaluation des performances éducatives.

Nous sommes issus tous les deux d'une expérience de recherche, en sociologie et en histoire des idées, orientée sur les déterminants culturels de longue durée qui expliquent l'originalité de l'expérience suédoise dans la réforme éducative. Votre biographie professionnelle rend votre point de vue essentiel pour reconstituer, pour les lecteurs de Nordiques, l'évolution récente de ce système qui, dans les comparaisons internationales, a souvent figuré comme une référence en termes d'inclusion et de performance. C'est pourquoi nous aimerions que vous nous aidiez à comprendre ce que cette entreprise a laissé comme traces durables. Nous allons ainsi remonter aux origines du changement d'orientation qui concerne les vingt-cinq dernières années et qui a fait de l'éducation une source d'inquiétude et de polarisation sur la scène publique suédoise. Pour ne mentionner que quelques enjeux sensibles, on peut évoquer les critiques formulées à l'encontre de la municipalisation dans les années 2011-2013, les difficultés à recruter des enseignants et, en réaction, le cycle des réformes mené par le ministre libéral du gouvernement de centre droit (2006-2014), Jan Björklund, proclamant la revanche de «l'école des savoirs " (kunskapsskola) sur une prétendue " école du flou » (fumskola). Les avis sont partagés sur le bilan de ces mesures, qui reviennent à un désaveu des fondements idéologiques des réformes antérieures.

3 Source : Skolverket.se/statistik, 2017.

4 Andreas Fejes, Magnus Dahlstedt, Skolan, marknaden och framtiden, Lund, Studentlitteratur, 2018.

5 La réforme de l'enseignement privé de 1992 comporta l'introduction d'un " chèque " ou voucher (skolpengen), à savoir une somme d'argent allouée par les municipalités à l'école de choix de chaque élève, en fonction du niveau de scolarité. Ce chèque accompagne l'élève lors de son passage d'un établissement à un autre, ce qui introduit un principe de concurrence entre les écoles.

6 Ulf P. Lundgren a été d'abord membre, puis président du comité de direction du Centre pour la recherche et l'innovation dans l'enseignement de l'OCDE. 
Nous allons donc nous concentrer sur la reconstitution historique du tournant des années 1990 et de son impact : sur la distance qui s'est creusée entre l'acteur étatique et la scène éducative. Mais nous souhaiterions placer le curseur encore plus loin. Cette expérience est pour nous l'occasion de sonder, avec votre aide, le rapport entre science, évolution du contexte socioculturel et organisation concrète du système - et entre facteurs structurels et conjoncturels du changement. Les faiblesses du système scolaire suédois sont-elles un reflet de mutations plus globales? Les constats de crise qui planent parfois sur l'école dans le monde développé (voire l'idée selon laquelle elle deviendrait de moins en moins pertinente pour les trajectoires des jeunes) ont-ils un fondement ? Quelle marge d'action est laissée aux décideurs politiques?

Piero Colla/Tomas Wedin : La transformation du système éducatif suédois à partir de l'après-guerre semble s'organiser selon trois cycles longs : un premier cycle qui s'étend de 1946 jusqu'aux années 1980 et se définit par la construction d'un système éducatif plus inclusif, égalitaire et intégré ; un deuxième cycle, entre 1989 et le début des années 2000, caractérisé par une dévolution des pouvoirs de l'État aux municipalités et dans une certaine mesure aux acteurs du marché ; puis un troisième cycle, lié à l'accession aux affaires du gouvernement de coalition de centre droit, Alliansen ("l'Alliance », 2006-2014). Cette phase se caractériserait par une révision critique d'éléments des deux phases précédentes, et par le rétablissement de certaines mesures : notation dès l'âge de 12 ans, séparation plus nette entre formations pratiques et formations théoriques, apparition dans les documents d'orientation de l'idée structurante de «l'esprit entrepreneurial $»^{7}$. Est-ce que vous partagez cette manière d'appréhender l'évolution du paysage éducatif ? Où situez-vous les points de fracture et la relation entre ces passages?

Ulf P. Lundgren : Dans les grandes lignes, je souscris à cette périodisation, même si on pourrait objecter qu'au niveau du secondaire (gymnasieskola), la réforme n'obéit pas aux mêmes logiques qu'au niveau de la grundskola. Le timing a été également différent : l'intégration du secondaire n'a débuté qu'à la fin des années 1960, dans un contexte différent par rapport au début de la réforme qui a mené à la grundskola, qui puise ses racines dans un autre contexte. Surtout, il est pertinent de rattacher ces trois étapes à des transformations sous-jacentes du contexte économique et productif. La première phase correspond clairement à un long cycle de croissance économique, qui, au niveau des institutions, s'exprime par une expansion constante du secteur public et, sur le plan psychologique, par un certain sentiment de stabilité, ou de prévisibilité. Pour visualiser ce contexte,

$7 \mathrm{Au}$ cours du dernier cycle de réformes, les curricula ont intégré la notion d' " apprentissage entrepreunarial " (voir Läroplanen för grundskolan 2011, Stockholm, Skolverket, 2011). Durant la préparation de ces réformes, le gouvernement Reinfeldt (centre droit) avait annoncé vouloir faire de ce concept un " fil rouge " parcourant l'ensemble du système éducatif (Regeringskansliet, Strategi för entreprenörskap inom utbildningsområdet, Stockholm, 2009, p. 9). 
on peut penser à la ville de Göteborg, avec ses chantiers navals en essor constant à l'époque.

À partir de la seconde moitié des années 1970, le paysage socio-économique change radicalement, entraînant une vision du rôle de l'État plus modeste que ce que le keynésianisme lui attribuait. L'idée de renforcer les responsabilités locales dans de nombreux secteurs de l'État providence et le débat subséquent sur la décentralisation du système éducatif interviennent dans ce contexte. C'est dès le début des années 1980, voire dans les débats préparatoires du $\operatorname{Lgr} 80^{8}$, qu'on commença à cultiver l'idée de gouverner le système sur la base de finalités générales, plutôt que de directives détaillées. Le projet de programme, qui remonte à la fin des années 1970, affirme clairement qu'il faut amener l'école dans cette direction. Mais ces idées ne seront développées qu'au moment de l'introduction de la notion de "compétences " à la fin des années 1980.

Un autre facteur à prendre en compte, pour comprendre la progression des réformes, est l'essor, au début des années 1990, des technologies de l'information et de la communication : l'accès universel aux ordinateurs et à la téléphonie mobile nous a plongés dans un monde différent, y compris du point de vue des formes de production. Cela impliquait que l'éducation était appelée à relever de nouveaux défis, car les compétences nécessaires aux individus sur le marché du travail ont radicalement changé.

Quant à la troisième phase, liée au tournant politique de 2006, elle doit être comprise sur fond du débat sur la mondialisation. Une autre circonstance cruciale a été, à mon avis, l'augmentation et l'accélération des flux d'information. Cela a donné à de nouveaux récits politiques la chance de se répandre beaucoup plus rapidement. Pour donner un exemple, une tension autour des réformes soutenues par les sociaux-démocrates existait déjà dans les années 1970, mais les arguments - aussi bien ceux des adversaires que des partisans de ces réformes - se fondaient en grande partie sur l'expérience directe des acteurs des controverses. Plus tard, à partir des années 1990 et 2000, les positions ont commencé à se polariser sur la base d'idées reçues, voire de fake news véhiculées par les médias. L'environnement de la politique éducative avait changé, et avec ce changement un nouveau type de décideur a émergé. On peut se demander si certaines des décisions qui ont été prises depuis 2006 l'ont été sur la base de résultats empiriques, qui justifiaient ces choix, ou seulement pour concrétiser un récit abstrait d'une école idéale. Prenez un politique comme Jan Björklund, que vous avez cité au début de l'interview : il a développé des thèses qui n'ont pas de véritable ancrage dans l'analyse scientifique des performances des établissements et des élèves. Je ne pense pas que cela aurait pu se produire dans les années 1950 ou 1960, lorsque le débat éducatif était rigoureusement fondé sur des résultats empiriques.

8 Läroplan för grundskola, Stockholm, Liber, 1980 (programmes pour l'enseignement obligatoire). 
P. C./T. W. : Durant le long cycle de croissance de l'après-guerre, et encore jusqu'aux années 1980, la réforme de l'école suédoise, à travers ses objectifs et ses solutions originales, a servi de source d'inspiration aux grands courants de réforme internationaux. Le nombre de voyages d'étude et de publications concernant la Suède, ainsi que certains rapports élogieux de l'OCDE', en portent la marque. L’originalité de cette expérience s'est sans doute estompée avec le temps, peut-être aussi parce que certains de ses traits caractéristiques se sont généralisés. Le retrait de l'État de son engagement traditionnel dans les politiques éducatives a été, en Scandinavie, un phénomène plus voyant qu'ailleurs. Des entreprises privées, et dans une certaine mesure la bureaucratie locale, sont devenues des acteurs à part entière comme responsables d'écoles, producteurs d'outils pédagogiques et de contenus, partenaires des enseignants et des familles. Estimez-vous qu'il existe encore une spécificité du modèle national, une " touche " suédoise dans l'aménagement du projet éducatif ? Où réside-t-elle, à votre avis ?

U. L. : Spontanément, je suis tenté de répondre que non. Ou plus exactement : cette spécificité concerne de nos jours bien plus la rhétorique que le contenu des politiques. Je me réfère à l'idéal de l'égalité, qui dans les débats suédois sur l'éducation continue d'avoir un poids supérieur à ce qui se passe dans les autres pays. À quelques exceptions près, bien sûr : en Norvège, par exemple, l'égalité - entre autres à cause de la tradition de politique régionale, qui pousse à contrecarrer les disparités économiques - se porte encore relativement bien, et l'expansion de l'enseignement privé a été très limitée.

P. C./T. W. : L'un des changements les plus importants de la fin du $\mathrm{XX}^{\mathrm{e}}$ siècle a été la réforme qui a donné aux familles une grande liberté de choix au niveau des parcours scolaires des enfants, en mettant public et privé sur un plan d'égalité et en favorisant le développement d'écoles privées subventionnées. Dans une perspective historique, il s'agit d'un tournant radical par rapport à la situation antérieure de quasi-monopole public. Comment analysez-vous la valfrihetsrevolution (" révolution de la liberté de choix ${ }^{10}$ ), le slogan que Carl Bildt a associé à cette réforme?

U. L. : Il s'agit de la construction progressive d'un champ de débats. Durant la seconde moitié des années 1980, j'avais participé, avec Olof Wennås et Lars Du Rietz, à une enquête parlementaire sur la gouvernance de l'école ${ }^{11}$. Cette demande s'inscrivait dans un débat international précédent et plus vaste, au sujet

9 Voir Reviews of National Policies for Education, Paris, OCDE 1969 et 1981.

10 Il s'agit du principe selon lequel chaque élève aurait le droit de choisir l'établissement scolaire - public ou privé - qui lui convient : un principe introduit en 1992-1993, puis renforcé par d'autres formes de déréglementation.

11 L'enquête fut mise en place en 1986. Voir Lars Du Rietz, Ulf P. Lundgren et Olof Wennås, Ansvarsfördelning och styrning på skolområdet, DsU 1987:1, Stockholm, Regeringskansliet, 1987 et SOU 1988:20 
de la démocratie et des prérogatives de l'État: en Scandinavie, cela a pris la forme des grandes enquêtes menées à cette époque sur l'état de la démocratie et la distribution des pouvoirs. La maktutredning, en Norvège, en a été le modèle. C'est dans ce contexte qu'il faut comprendre la montée, en Suède, d'un mouvement d'opinion en faveur d'un nouveau modèle de régulation de l'école. Deux positions se cristallisent alors : un courant, représenté tout d'abord par le parti libéral (Folkpartiet), puis par la droite (Moderaterna), réclamant bel et bien la privatisation de l'école, en confinant l'État dans une simple mission de contrôle ; l'autre courant, plus préoccupé de décentraliser les responsabilités.

La seconde stratégie s'inscrit dans la continuité de la politique précédente, et trouvait un terrain favorable en Suède, en raison du statut traditionnel des municipalités en tant qu'instance de codécision. Lorsqu'en 1989 la discussion sur le nouveau partage de compétences dans la gestion de l'école arriva au Parlement (Riksdag), les députés n’ont pas pu trouver un point de consensus ; les sociauxdémocrates étaient divisés, mais aussi bien le ministre de l'Éducation (socialdémocrate) que le parti de la gauche (Vänsterpartiet) s'opposaient à toute idée de privatisation, alors que les modérés et les libéraux souhaitaient aller vers un système de voucher pour l'enseignement privé. Le tournant, sur ce point, a donc été l'alternance au pouvoir entre sociaux-démocrates et centre droit en 1991.

P. $C . / T$. W. : D’après plusieurs études, la possibilité laissée à chaque élève de choisir son école, aggraverait la ségrégation - aussi bien sociale qu'ethnoculturelle - entre différentes écoles (de l'école primaire au lycée) : s'agit-il, d'après vous, d'un choix délibéré des classes sociales les mieux loties ? Avait-on anticipé que le système allait atteindre un tel niveau de fragmentation?

U. L. : Ce mouvement d'opinion, favorable à la création d'un " marché " éducatif, a rencontré un consensus politique très large, alimenté entre autres par des représentants illustres de la science politique. Beaucoup de tenants du "libre choix » ont réagi comme s'ils avaient eu une révélation! Ils croyaient sincèrement qu'il s'agissait de réformes indispensables pour améliorer l'état du système scolaire. C'était une vague montante : le tournant décisif a été le retour au pouvoir de la droite, en 1991, mais le nouveau climat avait aussi touché les sociauxdémocrates. Et le changement d'orientation s'est finalement imposé sans véritable opposition politique.

C'est comme cela qu'il faut expliquer la perte de contrôle de la déréglementation, le fait qu'on ait poussé les limites de plus en plus loin : on a mis un bateau à la mer et on a continué de le construire alors qu'il naviguait. Jusqu’à en arriver à une multiplication extrême des cours, des filières...

P. C./T. W. : Est-ce que cela n'est pas dû au fait que - si l'on fait abstraction de la dimension de la marchandisation - l'idée de centralité du niveau communal, de mettre "l'élève au centre » et de le laisser choisir, s'inscrivait dans la 
continuité du discours social-démocrate des années 1970 et 1980 ? On a reproché aux sociaux-démocrates d'avoir "viré à droite ", mais n'y a-t-il pas là une forme de cohérence, plutôt qu'une rupture?

U. L. : Je crois que oui, il y a en effet des traits de continuité. Mais on a également assisté à l'incorporation d'arguments purement libéraux dans l'argumentaire des sociaux-démocrates.

P. C./T. W. : Nous voudrions maintenant vous interroger sur des aspects structurels des réformes, où l'expérience suédoise peut receler des leçons. Durant votre expérience, d'abord au sein de l'OCDE, puis comme directeur de Skolverket, vous avez été témoin d'une transformation très profonde, qui touche au critère même de gouvernance des systèmes éducatifs occidentaux : le passage à la notion de "compétence ", doublé d'une organisation plus flexible des missions des enseignants, axée sur des objectifs à atteindre (målrelaterad). L'expression de ce tournant en Suède a été les réformes de décentralisation, entreprises entre 1989 et le début des années 1990. L'État décida alors d'abandonner les programmes détaillés du passé, de limiter la gestion centrale à l'énoncé d’objectifs généraux dont les acteurs doivent assurer la réalisation, en puisant dans les moyens - corpus, méthodes, dotation horaire par sujet et matière... - les plus adéquats. Quels ont été les sources d'inspiration et les espoirs à l'origine d'un tel changement de registre?

U. L. : L'émergence de la notion de "compétences" tient au sentiment que l'avenir est devenu plus incertain : c'était aussi l'une des conséquences de la crise économique des années 1970, et des crises qui se sont succédé depuis lors. Le futur est devenu de plus en plus difficile à décoder, à anticiper. Un sociologue suisse, Walo Hutmacher, a été tout particulièrement influent au début des années 1990 : à travers ses ouvrages, il montrait que le changement technologique ouvre des possibilités qui rendent complètement impossible l'anticipation des connaissances nécessaires aux générations de demain. L'enseignement devrait donc se concentrer sur la compréhension de concepts généraux, susceptibles de se traduire dans les formes les plus imprévisibles. Bien sûr, cette difficulté était présente auparavant, mais le rythme du changement, depuis les années 1960, s'est accéléré. Ainsi, au début des années 1990, on a émis l'idée suivante : puisqu'il est impossible d'anticiper les connaissances qui seront indispensables dans le futur, on devrait focaliser les efforts sur l'aptitude des élèves à se procurer celles dont ils auront besoin au moment voulu.

Ensuite, l'idée des compétences a eu un rôle central lors du premier classement PISA $^{12}$, en 2000. J'y étais moi-même favorable, en tant que stratégie permettant

12 Le programme PISA est un ensemble d'études pour mesurer les performances des systèmes éducatifs et concerne toutes les matières enseignées. 
de contourner un problème qui avait été une cause d'insatisfaction au sein de l'OCDE : à savoir la pertinence des classements internationaux des performances scolaires, dressés dans le cadre de l'IEA ${ }^{13}$. On avait pris conscience que les paramètres utilisés ne facilitaient pas les rapprochements des données, car celles-ci étaient comprises différemment selon les contextes et les cultures locales. C'est alors que le concept de compétence s'est imposé, comme instrument pour affiner la fiabilité des enquêtes. Reste cependant une question de fond, à savoir : une fois qu'une autorité nationale a fixé les concepts, qui se chargera de déterminer les connaissances concrètes à aborder dans l'enseignement ? À la base de la réforme de 1994 se trouvait l'idée qu'il incombait aux professeurs, sur le terrain, de concrétiser le läroplan (" plan d'enseignement ») - d'éviter qu'il soit réduit à une seule dimension, une seule lecture.

P. C. /T. W. : Quelle a été la part respective, dans ce processus de déréglementation, des facteurs structurels et économiques, d'une part, et des causes endogènes, d'autre part ? Vous avez vous-même remarqué que ces réformes avaient des précédents éloignés, dans le débat qui avait accompagné la création de réseaux internationaux d'évaluation pour améliorer les performances des systèmes éducatifs en Europe occidentale ${ }^{14}$.

U. L. : Les facteurs économiques externes jouent un rôle bien plus structurant dans l'histoire de l'école que celui habituellement attribué par les historiens de l'éducation, lorsqu'ils décrivent l'avènement et le succès des différents courants pédagogiques. Il est donc crucial de prêter attention aux conditions d'existence des théories pédagogiques : quand on énonce les connaissances qu'il est essentiel de transmettre, par exemple, il n'est pas indifférent de savoir si c'est l'État qui prend l'initiative et définit le cadre, ou les forces du marché. Une question qui mérite d'être posée consiste à savoir dans quelle mesure la décentralisation des responsabilités change le cadre et la nature de la production des objectifs pédagogiques. On n'en est pas encore là, car jusqu'à présent, l'État garde toujours une certaine emprise - par ses procédures d'inspection et ses programmes nationaux - sur le système éducatif.

En même temps, il y a d'autres constantes que l'on pourrait qualifier d' " endogènes " : nous assistons au retour périodique, dans l'histoire du système éducatif suédois, d'un nombre relativement restreint de "codes " ${ }^{15}$. L'un d'entre eux consiste à considérer les connaissances comme une entité tangible et mesurable, concept qui revient régulièrement.

13 International Association for the Evaluation of Educational Achievement, crée en 1958.

14 Ulf P. Lundgren, "What's in a name ? That which we call a crisis ? A commentary on Michael Young's article "Overcoming the crisis in curriculum theor" ", Journal of Curriculum Studies, 47.6, 2015, p. 787-801.

15 Voir Ulf P. Lundgren, Att organisera omvärlden. En introduktion till läroplansteori, Stockholm, Liber, 1979 . 
P. C. /T. W. : Passons à un outil de réforme que vous avez contribué à analyser à travers la théorie du "facteur-cadre " (ramfaktor), une approche développée par vous et vos collègues Urban Dahlöf et Jarl Bengtsson à la fin des années 1960 : à savoir les programmes d'enseignement. Depuis les années 1990, on constate une évolution vers des curricula moins détaillés, qui laissent aux acteurs une large marge d'interprétation et de choix du matériel d'étude. Quel a été le rôle de la recherche - et de la théorie pédagogique - dans l'évolution vers cette nouvelle direction?

U. L. : J'aimerais rappeler l'origine du concept de «facteur-cadre ». L'idée avait été développée par mon ami Urban avec une portée limitée : elle visait la question de la différenciation des filières ${ }^{16}$, que l'introduction de la grundskola allait progressivement supprimer. Par la suite, j'ai développé cette idée comme un outil de planification. Je me suis efforcé d'échafauder un modèle d'analyse qui prenne en compte le cadre, à savoir les conditions objectives pour que la planification éducative - par exemple l'établissement d'un programme - soit efficace, et pour conjurer les échecs. Ce modèle a eu un certain écho au niveau international, mais une diffusion limitée en Suède.

Si l'on aborde la question sur un plan plus général, les premières décennies après la guerre ont été caractérisées par un fort consensus - notamment chez les sociaux-démocrates - autour de l'idée que la politique éducative et l'écriture des programmes devraient évoluer selon des schémas rationnels, reposant sur des bases scientifiques. Un exemple parfait se trouve dans les analyses du professeur Härnkvist - qui fut mon directeur de thèse - de la " réserve de talents " que l'école obligatoire devait s'efforcer de valoriser. De telles études, qui proposaient une explication objective des causes de l'échec scolaire, ainsi que d'autres relatives aux conditions de l'apprentissage du suédois, ou des mathématiques - et qui proposaient des solutions didactiques plus inclusives - ont eu un impact politique immédiat. Ces chercheurs avaient un accès direct aux ministres de l'époque, notamment Olof Palme.

L'une des choses qu'on me faisait remarquer, lorsque je m'entretenais avec des collègues étrangers, est justement le fait que les réformes éducatives en Suède étaient fondées sur des résultats scientifiques. Je dirais que cet héritage a été délaissé au début des années 2000. En conséquence de l'accélération des processus décisionnels, le système des commissions d'enquête (utredningar) ne peut plus jouer son rôle comme autrefois, alors qu'il était un point de rencontre fondamental entre l'univers savant et la politique. Les nouvelles autorités centrales

16 Avant la réforme de 1962, qui a introduit un seul tronc commun de formation obligatoire, les élèves se partageaient entre plusieurs filières parallèles, d'après un modèle comparable à celui de l'Allemagne aujourd'hui. 
qui ont vu le jour dans les années 2000, comme l'Institut de recherche sur l'école (Skolforskningsinstitutet), n'ont pas rempli ce vide. De nos jours, les décideurs politiques demandent une seule chose à la recherche pédagogique : qu'elle confirme leurs propres convictions. Certains politiques me l'ont dit ouvertement.

P. C./T. W. : Portons maintenant notre attention sur la situation des acteurs du système. D’après les intentions affichées dès 1991, les enseignants étaient censés être les grands bénéficiaires de la délégation du pouvoir du centre au niveau territorial. Cependant, cette approche ne semble avoir abouti ni à légitimer les réformes aux yeux des enseignants ni à augmenter leur sentiment de satisfaction. Au contraire, depuis une vingtaine d'années, les statistiques relèvent chez les professeurs une vision pessimiste de la considération sociale accordée à leur profession, alors que la pression pour accéder aux filières de formation a fortement diminué au cours des vingt dernières années. Quelles sont les causes de cette désaffection?

U. L. : Le but des réformes des années 1990 était d'augmenter l'influence des professeurs sur le système. Mais la crise économique est intervenue au début de la décennie, mettant fin aux discussions que nous aurions aimé voir surgir autour de ces questions. Par ailleurs, des contraintes financières ont empêché aussi bien l'État que les communes d'accorder l'aide, en termes de formation continue, qu'ils auraient été obligés de fournir. En conséquence de l'intensification des critiques et des signaux d'alarme sur la situation de l'école, l'État a endossé de nouveau une posture interventionniste - notamment sous les gouvernements de centre droit (2006-2014). Toutefois, au lieu de revenir à une approche systémique, il s'est attaché à " boucher les trous ", avec des mesures ciblées qui contraignent souvent les acteurs et ont pour résultat de limiter leur marge d'action. Il ne faut pas s'étonner que ces réformes, nées dans un climat de méfiance à l'égard de ceux qui étaient censés les mettre en place, aient finalement échoué.

P. C./T. W. : Il est légitime de s'interroger sur l'évolution du métier d'enseignant au cours de cette période. Du fait des réformes des années 1990, la part des tâches organisationnelles et bureaucratiques dans l'emploi du temps s'est accrue. Ensuite, la réforme de la formation des enseignants de 2001 a mis en avant une figure de professeur " généraliste ", dont la formation spécialisée et la préparation de type disciplinaire ont perdu en importance. Depuis quelques années, l'État a pris des mesures dans le sens contraire ; comment expliquez-vous, cependant, le paradoxe entre les tentatives de mieux définir fonctions et tâches de l'enseignant et un tel sentiment de perte de prestige?

U. L. : La Suède a connu un grand nombre de réformes qui touchent à la mission des professeurs - il suffit de penser à celles qui ont concerné la notation 
des élèves, qui se sont succédé à une fréquence élevée. Et puisque, dans beaucoup de cas, ces mesures impliquaient pour l'enseignant des charges plus lourdes - formulaires à remplir, tâches administratives, etc. -, une lassitude s'est installée. Il y a notamment une tendance attachée aux théories de la nouvelle gestion publique (New Public Management) et qui prévoit une description de plus en plus méticuleuse de chaque aspect du travail, des protocoles à respecter... bref, des obligations qui coûtent souvent plus qu'elles ne rapportent. Cette tendance a fortement contribué au mécontentement des enseignants. L'écart entre la volonté de renforcer le rôle des enseignants et leur perception subjective s'est donc creusé.

P. C./T. W. : Quel est votre diagnostic sur l'état de la formation des enseignants ? Les dernières réformes ont-elles été à la hauteur des difficultés que vous venez d'évoquer?

U. L. : Disons pour commencer que les conditions d'admission aux formations des professeurs varient selon les établissements, et il y a ceux qui attirent plus d'étudiants qu'ils ne peuvent en accepter. Une première question à se poser est donc la raison pour laquelle certaines formations sont plus attrayantes et d'autres échouent.

Il me semble que beaucoup de formations pour enseignants sont attachées à une tradition bien spécifique. Il s'agit d'une culture, disons, peu académique, puisant ses racines dans l'ancien cursus des enseignants de la folkskola (les seminarier ${ }^{17}$ ) : des établissements dominés par la plus grande méfiance vis-à-vis de la tradition académique des professeurs du secondaire, le läroverk. Ces écoles constituaient une sorte de cercle intime, lié à un style pédagogique particulier, ce qui fait par exemple que l'on s'adresse aux enseignants comme s'ils étaient à leur tour des écoliers dans une classe. Dans cette tradition, d'après mes souvenirs, l'exigence de fonder son enseignement sur une base scientifique était plutôt faible. Pour bien comprendre ce clivage avec le monde de la recherche, il faudrait effectuer un travail d'anthropologue.

P. C./T. W. : Revenons sur le véritable " choc" qu'a accompagné, en Suède, le débat sur les résultats des tests PISA en $2013^{18}$. La réaction des politiques fut alors que la décentralisation était allée trop loin, que l'État devait " resserrer les boulons ». Néanmoins, les mesures prises depuis la fin des années 1990 allaient déjà dans le sens d'un contrôle central plus fort. Ainsi, le corps d'inspection a été

17 À partir de lintroduction de l'enseignement primaire obligatoire (1842), les folkskoleseminarier - institués au niveau des municipalités et sous lrautorité de l'Église - ont assuré la formation des enseignants du premier cycle de scolarité.

18 En décembre 2013, les résultats du classement PISA avaient révélé un déclin des performances des élèves suédois en lecture, mathématiques et sciences plus fort que dans n'importe quel autre pays de l'OCDE (https://www.svd.se/pisachocken-varst-i-valmaende-lander). 
restauré et ensuite renforcé par la création d'une agence (2008), le rôle managérial des proviseurs a été accentué, etc. Ne serait-il pas plus correct de penser qu'entre 1990 et nos jours, les contraintes qui pèsent sur l'enseignant ont augmenté, tout en changeant de forme et d'objet ? D'après le sociologue Ulf Lundström, l'incitation à l'autonomie est, dans les nouvelles formes de gestion, intrinsèquement contradictoire ${ }^{19}$. Cette dynamique, à votre avis, laisse-t-elle encore un futur à l'école en tant qu'institution autonome, attachée à des finalités qui lui sont propres?

U. L. : J'aimerais que ce soit le cas : l'école retrouverait alors sa place dans la société - à la fois en tant que force de critique et de transformation. Ce qui me fait peur, c'est la bureaucratisation croissante et la manière dont l'école se trouve de plus en plus sous l'emprise d'instances de contrôle, à commencer par l'État : certaines décisions devraient être prises au niveau le plus proche, pour que ceux qui décident soient informés des conditions dans lesquelles les acteurs œuvrent.

P. C./T. W. : Mais au fond la question est : qui a pris le contrôle de l'école ? Selon quelle stratégie ? Les " compétences " qui occupent une place de plus en plus centrale concernent, le plus souvent, des qualités exigées par le marché du travail (l'esprit d'entreprise, par exemple), ou se fondent sur des demandes un peu abstraites d'ajustement à l'air du temps. L'ancrage des politiques scolaires dans le présent, la forme de gouvernance « réactive » que vous avez dénoncée dans l'un de vos écrits, semble être devenu une condition incontournable. Y a-t-il, à votre avis, des possibilités de réintégrer dans les outils de direction du système les dimensions temporelles que l'école a perdues de vue, à savoir le futur et le passé - un passé qui fonctionne, depuis la fin des années 1960, comme une sorte d'antimodèle dans les rhétoriques de la réforme scolaire ${ }^{20}$ ? La renaissance de la bildning ${ }^{21}$, qu'avec votre collègue sociologue Donald Broady vous aviez placée au centre d'une célèbre enquête publique, en 1991, n'était-elle pas une tentative de sortir de l'impasse ${ }^{22}$ ?

U. L. : La réflexion qui nous a inspirés était la suivante : ce qui caractérise le développement des systèmes éducatifs, à partir de 1900, a été la notion d'utilité. Les élèves devraient être instruits en connaissances susceptibles de générer des profits dans le futur. Or, à partir du moment où il devient impossible de prédéterminer quelles connaissances vont être effectivement nécessaires, on pourrait laisser de côté l'exigence utilitariste, et remettre en jeu un type de connaissance qui soit stimulante, qui permette de renouer un contact avec un héritage culturel,

19 Ulf Lundström, "Teacher autonomy in the era of New Public Management ", NordSTEP, 1, 2015.

20 François Hartog, Régimes d'historicité, Paris, Seuil, 2012.

21 Formation générale ou classique (dans un sens proche de la notion de Bildung, en allemand).

22 SOU 1992:94, Skola för bildning, Stockholm, Statens offentliga utredningar, 1992. 
avec le passé. Ce qui peut-être n'est pas utile au sens étroit du terme, mais l'est dans une acception plus globale - du point de vue de la constitution d'une identité, d'une place dans le monde. C'est peut-être à travers la notion de « formation de la personnalité ", laquelle autrefois a été centrale dans la théorie de la réforme éducative, que l'on peut fournir aux élèves le meilleur outillage pour affronter l'avenir. Ce qui caractérise les élèves qui étaient issus des formations classiques, est justement qu'ils n'y ont pas appris comment faire les choses, mais qu'on peut le faire, qu'on doit le faire : que l'on se doit d'essayer. Mais l'idée de la formation de la personnalité a complètement disparu du débat scolaire. Personne n'ose plus la convoquer, de peur d'être considéré comme un tenant de la flumskola, de l'école du «n'importe quoi ».

P. C./T. W. : À côté de l'utilité, nous avons décelé à travers nos recherches des éléments de continuité entre les différentes phases de la réforme scolaire que nous venons d'aborder. La place de l'individu, par exemple, n'a cessé de s'accroître : le système suédois se caractérise par l'absence d'examens, la liberté de choisir son école et ses disciplines, mais aussi par la liberté des enseignants par rapport aux canons disciplinaires, et des gestionnaires locaux par rapport à l'État. Ce qui semble absent du tableau est peut-être un cadre commun : un rappel de la fonction sociale de l'éducation, sa raison d'être pour des sociologues comme Émile Durkheim. Lors de la révision des programmes, en 1991-1994, vous aviez baptisé ce fonds commun "valeurs fondamentales " (värdegrund), en l'associant essentiellement aux principes démocratiques. Le mot, depuis lors, a eu du succès : l'énoncé du värdegrund trône en tête des programmes scolaires. On voudrait vous demander si ce ciment imaginaire a joué la fonction que vous lui avez attribuée, et si vous estimez qu'il est suffisant pour rendre à l'éducation un sens, du point de vue social ?

U. L. : Dans les programmes de 1994, nous avions défini la notion de värdegrund comme un idéal qui devrait œuvrer à tous les niveaux d'activité de l'école : la base (grund) sur laquelle les acteurs interviennent, non l'objet d'une pédagogie, quelque chose dont il faut parler, mais qu'il faut " pratiquer " constamment, selon la notion classique des pédagogies fondées sur l'activité. Par la suite, le concept a été absorbé et dilué dans différents contextes ; avec sa transfiguration pédagogique, sa transformation en un objet de savoir - au point que des universités ouvrent des chaires de värdegrund -, son contenu original s'est perdu.

P. C. /T. W. : On voudrait terminer en insistant sur une dimension du processus d'individualisation qui semble indissociable, dans la culture éducative suédoise, de la notion de "formation de la personnalité " que vous évoquiez auparavant. Depuis les années 1940, l'importance d'adapter l'enseignement aux conditions locales, à l'horizon d'expériences de l'élève en tant qu'individu, a été un objectif 
central des initiatives de réformes. N'y a-t-il pas un risque que cet accent sur un horizon immédiat, perceptible, devienne un frein ? Que l'on finisse par enfermer les élèves dans une prison présentiste, au lieu - pour se référer aux thèses classiques de Hannah Arendt - de s'intercaler entre leur monde et le monde, qu'ils ne connaissent pas $^{23}$ ?

U. L. : Vous avez complètement raison, ce risque est bien présent - nous nous enfermons dans l'ici et maintenant. Il suffit de considérer la prose des rapports d'enquêtes : de 1946 (lorsqu'un poète, comme Stellan Arvidsson, pouvait diriger les travaux) à nos jours, l'horizon intellectuel s'est restreint. La langue est devenue plus aride, mais l'ambition et la perspective chronologique des documents se sont aussi faites plus modestes. Avec le Läroplanskommitté, en 1991, Donald Broady, qui est un écrivain raffiné, Berit Hörnqvist, très attentive à la qualité de l'expression, et moi-même, nous avions cherché à introduire une note, un style différent. C'était l'expression de notre ambition pour l'école.

\section{Propos recueillis par Piero Colla ${ }^{24}$ et Tomas Wedin ${ }^{25}$}

23 Hannah Arendt, La crise de la culture, Paris, Gallimard, 1972.

24 Chercheur associé auprès du centre Maurice-Halbwachs (CNRS-EHESS-ENS); professeur de didactique de l'histoire à l'université de Bologne.

25 Doctorant en histoire des idées à l'université de Göteborg. 